\title{
E-learning appliqué à la formation viticole : principe, exemples des projets E-VitiClimate et Ecowinery
}

\author{
Joël Rochard ${ }^{\mathrm{a}}$ \\ IFV (Institut Français de la Vigne et du Vin), Pôle National Développement Durable, 17 rue Jean Chandon Moët, \\ 51200 Epernay, France
}

\begin{abstract}
Résumé. La formation en ligne est une des technologies de l'information et de la communication pour l'éducation, intégrée dans la cyberculture. Elle permet de s'affranchir, au moins partiellement, de la présence physique d'un enseignant à proximité. En revanche, le rôle du tuteur distant peut-être envisagé, avec un rôle de facilitateur et de médiateur. Le vocable E-learning évoque une synergie nouvelle entre des pratiques pédagogiques et des technologies éducatives permise par l'explosion d'Internet depuis les années 2000 et son immense potentiel de transfert mondial des connaissances. Le E-learning nécessite un investissement d'expertise scientifique, pédagogique et informatique initial avec un certain coût. Mais au-delà d'un seuil d'apprenants, le coût réel de la formation devient rapidement inférieur par rapport aux méthodes académiques. L'E-learning utilise notamment les pédagogies actives et didactiques par le jeu en liaison éventuellement avec un autocontrôle. Celui-ci peut être associé à une reconnaissance validée par un certificat. Pour les plates-formes gratuites l'accès est peu coûteux, l'apprentissage peut s'exercer à domicile (faible impact carbone) avec une flexibilité de la gestion du temps et la possibilité d'apprendre à son propre rythme tout en permettant par des liens, et éventuellement une plateforme ressource, d'approfondir les connaissances jusqu'au niveau souhaité par chaque étudiant. Au-delà de la diversité des langues utilisées, il est par ailleurs possible d'adapter l'apprentissage à un contexte spécifique (spécificités locales et climatiques, type d'itinéraire ou de process etc.). Les projets E-VitiClimate www.eviticlimate.eu et Ecowinery www.ecowinery.eu s'inscrivent dans le cadre du programme européen Léonardo Da Vinci et offrent une plate-forme innovante pour contribuer à une approche durable contribuer à une approche durable des vignobles européens. Les apprenants (professionnels, étudiants, conseillers, chercheurs, etc.) peuvent ainsi accéder à des modules de formation en E-learning et différents outils pédagogiques. E-VitiClimate vise à relever les enjeux viticoles que pose le changement climatique (origine, impacts et adaptation) tandis qu'Ecowinery est en liaison avec une conception écologique des caves (eau, énergie, intégration paysagère). Les principaux atouts de ces plates-formes pédagogiques sont les suivants : Un visuel simple d'utilisation, très illustré pour un apprentissage ludique avec des explications d'exemples complexes réalisées de façon simple et claire. Des informations modulables, en cascade, en fonction du degré d'enseignement choisi. Une série d'exercices et un test final par module, permettant l'obtention d'un certificat. Des vidéos en ligne avec l'avis des experts et des professionnels sur la thématique. Une documentation accessible aux apprenants sur les différents thèmes abordés. Une plate-forme ressource dotée d'une bibliographie très fournie. Les supports d'E-learning ne visent pas à remplacer les différentes fonctions de l'école (vie sociale, processus d'apprentissage collectif, échanges directs avec l'enseignant, etc.). Néanmoins, pour des sujets émergeant, à l'image du développement durable, ce type de support peut permettre à un large public d'accéder à des connaissances nouvelles, qui ne sont pas toujours disponibles dans les structures d'enseignement académique. Ce type de démarche pourrait être encouragé par l'Organisation Internationale de la Vigne et du Vin dans le prolongement du parrainage des congrès scientifiques internationaux. Un effet relais pourrait également être assuré au travers d'un portail didactique international multi langues en lien avec le site de l'OIV.
\end{abstract}

\begin{abstract}
Online training is one of the technologies of information and communication for education, integrated into internet. It eliminates, at least partially, the physical presence of a teacher nearby. However, the role of remote tutor may be considered, with a facilitator and mediator. E-learning word evokes a new synergy between teaching practices and educational technologies enabled by the Internet explosion since 2000 and its huge potential global knowledge transfer. E-learning requires an investment of scientific expertise, educational and initial data with a cost. But beyond a threshold of learners, the actual cost of the training is fast becoming lower in relation to academic methods. E-learning in particular using active pedagogies and learning through play in conjunction with a possibly self. It may be associated with a recognition validated by a certificate. For free access platforms is inexpensive, learning can take place at home (low carbon) with a flexible time management and the ability to learn at their own pace while allowing by links, and possibly a resource platform to deepen the knowledge to the desired level by each student. Beyond the diversity of languages, it is also possible to adapt
\end{abstract}

\footnotetext{
${ }^{\text {a }}$ Auteur de correspondance : joel.rochard@vignevin.com
}

This is an Open Access article distributed under the terms of the Creative Commons Attribution License 4.0, which permits unrestricted use, distribution, and reproduction in any medium, provided the original work is properly cited. 
learning to a specific context (local climate and specificity, route type or process etc.). Projects E-VitiClimate www.eviticlimate.eu and Ecowinery www.ecowinery.eu from the European program Leonardo da Vinci, and offer innovative platform for the sustainability of European vineyards. Learners (professionals, students, consultants, researchers, etc.) can access to training modules in E-learning and different educational tools. E-VitiClimate aims to raise the wine sector issues posed by climate change (original, Impacts and Adaptation) while Ecowinery is in connection with an ecological design cellars (water, energy, landscaping). The main advantages of these educational platforms are: A simple visual use, highly illustrated for playful learning with explanations of complex examples carried out simply and clearly. Modular information, waterfall, depending on the chosen level of education. A series of exercises and a final test module for obtaining a certificate. Videos online with the experts and professionals on the theme. Accessible documentation learners on different topics. A resource platform with a well-stocked bibliography. The E-learning materials are not intended to replace the various school functions (social, collective learning, direct interaction with the teacher, etc.). However, for emerging issues, like sustainable development, this type of support can allow a wider public access to new knowledge, which is not always available in the academic education structures. This type of approach could be encouraged by the International Organization of Vine and Wine in the extension of the sponsorship of international scientific conferences. A relay effect could also be provided through an international multi-language educational portal in connection with the OIV site.

\section{Introduction}

Le vocable E-learning associe une synergie nouvelle entre des pratiques pédagogiques et des technologies éducatives innovantes permises par l'explosion d'Internet et son potentiel de diffusion auprès d'un large public. L'union européenne définit l'outil le E-learning comme "l'utilisation de nouvelles technologies multimédia de l'internet pour améliorer la qualité de l'apprentissage en facilitant par l'accès à des ressources et des services et d'autre part des échanges et la collaboration à distance». Cette méthode de formation/éducation permet au moins en partie de s'affranchir de la présence physique d'un enseignant à proximité. En revanche, un tuteur distant peut intervenir avec des activités de facilitateur et de médiateur. Ce concept permet à l'apprenant de suivre la formation entièrement à distance mais peut parfois bénéficier d'un tutorat par des outils de type «web conférence », forum, chat, téléphone, courriel. Selon la formule choisie, la formation peut être suivie à n'importe quel moment, à n'importe quel rythme et depuis n'importe quel endroit.

Cette méthode d'apprentissage en ligne a d'abord majoritairement concerné la formation continue dans le prolongement des supports papier classiquement utilisés dans la formation à distance avec la possibilité de bénéficier d'un enseignement plus personnalisé adapté à différents niveaux, l'enseignement supérieur puis la formation en entreprise. Mais progressivement l'outil E-learning a concerné un public plus large : formation en entreprise, enseignement supérieur et formation initiale.

\section{Caractéristiques du E-learning}

La première étape de la mise en place d'un projet d'E-learning repose sur la création d'un cahier des charges qui intègre notamment les points suivants :

- domaine d'activité de l'outil,

- profil du public ciblé,

- raisons du développement du projet,

- ressources disponibles,

- objectifs poursuivis et avantages attendus,

- personnes et équipes responsables de la gestion du projet.
Les outils d'E-learning recouvrent généralement deux catégories de contenus :

- Contenus synchrones : ils nécessitent que l'apprenant et le formateur soient connectés à la plateforme en même temps (classe virtuelle, chat, vidéoconférence).

- Contenus asynchrones : l'apprenant y accède à tout moment. Il peut mettre en pause et répéter sa séquence d'apprentissage tout en mobilisant par un processus interactif des informations complémentaires (documents, schémas, animations, vidéos, liens internet, etc.).

D'un point de vue pratique la solution d'E-learning intègre deux volets opérationnels indépendants :

- la plateforme technique réalisée par une société informatique spécialisée.

- le contenu pédagogique avec des contenus standards éventuellement personnalisés ou contextualisés.

En plus des outils pédagogiques, l'interface informatique entre le formateur et les apprenants peut utiliser différents moyens de communication :

+ Messagerie : système de communication décalé, utilisé le plus souvent d'une seule personne à l'autre ;

+ Forums : système de communication asynchrone où tous les participants au forum peuvent suivre toutes les discussions ;

+ Chat ou messagerie instantanée : système de communication synchrone (c'est-à-dire en simultané) permettant à plusieurs personnes de discuter en temps réel ; + Audio ou vidéoconférence : chaque participant équipé d'un micro et d'une caméra peut prendre part à la vidéoconférence. C'est le moyen de communication le plus proche d'une interaction en présentiel ;

+ Tableau blanc interactif : il peut s'agir d'un tableau interactif réel, utilisé par le formateur lors de sessions vidéo et utilisable à distance. Il peut aussi s'agir d'une zone partagée dans le navigateur sur laquelle les utilisateurs peuvent échanger des schémas ou textes rapides ;

+ Documents partagés : un dépôt de documents partagés permet de distribuer des supports structurés à une partie ou à tous les apprenants ;

+ Wiki : site web librement éditable ;

+ Sondages : ils permettent de recueillir l'avis des apprenants sur les contenus, les dates, les fonctionnalités de la plateforme ; 
+ Calendrier et agenda : ils servent à définir des dates butoir pour certains événements (évaluations, sessions présentielles etc.).

La préparation des contenus pédagogiques représente la part la plus importante et la plus sensible dans la mise en place d'une plateforme E-learning. Il est souvent nécessaire de remettre en cause la forme et la structure des formations classiques et de les adapter dans une forme didactique et interactive. Il est par exemple possible d'importer des fichiers PowerPoint avec leurs notes et d'y ajouter des narrations et des documents annexes, ou saisir l'occasion pour réécrire les supports pour les reformater sous forme de modules E-learning dans un contexte plus interactif et multimédia (vidéos, quizz, jeux). Il est important d'intégrer qu'une session de formation en ligne, sans formateur présent, doit absolument être adaptée, enrichie en fonction des capacités et du profil des apprenants visés.

Pour motiver les apprenants il est éventuellement possible de créer des 《 serious games» (ou « jeux professionnels ») qui tirent parti des technologies du jeu vidéo dans un but pédagogique. En effet l'assimilation est la mémorisation d'un savoir, d'un savoir-faire, sont favorisés par le contexte environnemental dans lequel se situe la situation d'apprentissage. La conception de « serious games » nécessite souvent de privilégier l'aspect pratique de la formation et l'apprentissage par l'expérience.

Ils peuvent prendre plusieurs formes, des plus simples au plus évoluées :

- quiz avec une composante ludique ;

- jeu de rôle ou d'aventures : l'apprenant interagit avec des avatars ou son environnement pour mener à bien une mission ;

- simulation de situation réelle : geste technique, situation d'urgence.

\section{Retour d'expérience de deux projets européens : E-VitiClimate et Ecowinery}

Les projets E-VitiClimate et Ecowinery www.ecowinery.eu s'inscrivent dans le cadre de projets européens Léonardo Da Vinci en lien avec la formation initiale et l'enseignement professionnel (Fig. 1).

E-VitiClimate [1] www.eviticlimate.eu est une ressource éducative en ligne développée pour :

- Informer tous les acteurs du monde du vin, y compris ceux impliqués dans la production, l'éducation et la recherche, sur les défis, les menaces et les opportunités que représente le changement climatique pour les producteurs de vin en Europe.

- Identifier les stratégies qui pourraient être utilisées par les gestionnaires des vignobles et des chais, afin de s'adapter aux changements climatiques.

La structure de l'outil repose sur deux modules d'apprentissage sur le changement climatique et la production vinicole composé de contenu éducatif, d'exercices et de tests

- Module 1 : Vue d'ensemble des changements climatiques et de la production vinicole.

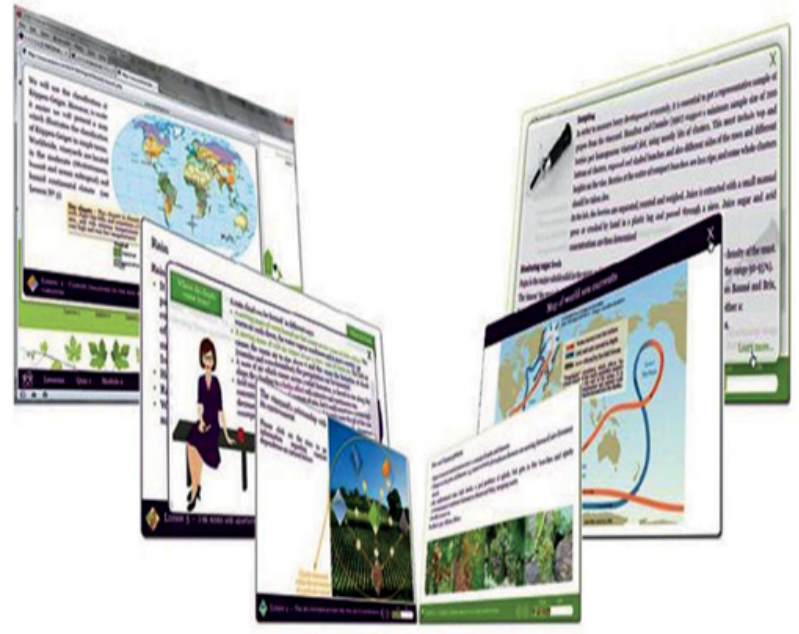

Figure 1. Aperçu des supports pédagogiques du projet.

- Module 2 : Aperçu régional du changement climatique et de la production vinicole.

Parallèlement une plateforme ressource donne accès à des contacts utiles, des publications, un glossaire et des ressources pour ceux qui souhaitent compléter leur apprentissage.

Le contenu éducatif couvre un large éventail de sujets qui permettent d'expliquer la relation importante et parfois complexe entre le climat, la viticulture, la production vinicole et la durabilité. Les principaux sujets traités sont les suivants :

- l'origine et le rôle des saisons, la météorologie et le climat ;

- l'histoire du climat, le changement climatique et les prévisions climatiques ;

- les indices bioclimatiques, la sélection des variétés cultivées, les techniques de vinification et styles de vins ;

- le terroir, la culture du vin et l'approche paysagère.

Le premier module présente le changement climatique global, les effets sur les vignobles européens et les pistes d'adaptation. Il contient des informations générales avec des exemples spécifiques : un panorama mondial du sujet, des observations réalisées au cours des 40 dernières années, les scénarios du Groupe d'experts intergouvernemental sur l'évolution du climat (GIEC). En complément l'outil développe l'évolution de la viticulture et la production de vin, les éléments de réflexion concernant les adaptations possibles au vignoble et à la cave, ainsi que les enjeux au niveau des sols, des ressources hydriques, des gaz à effet de serre, des températures, des pathogènes, et les éléments naturels influençant les climats locaux (latitude, altitude, inclinaison, etc.).

Parallèlement les méthodes de caractérisation climatique les zones viticoles sont décrits, permettant de mieux comprendre les phénomènes climatiques et leurs impacts potentiels depuis les raisins jusqu'au vin.

+ Impacts sur la vigne : précocité des stades phénologiques, augmentation de la photosynthèse, avancement des dates de vendanges, déséquilibre sucre/acide 
dans les raisins, accentuation du stress hydrique, développement de nouveaux parasites par modification des écosystèmes viticoles, etc..

+ Impacts sur le vin : augmentation du degré alcoolique, diminution de l'acidité et de la capacité de vieillissement des vins, oxydation prématurée des vins blancs, augmentation de la variabilité des millésimes, etc.

Le deuxième module reprend les différents aspects développés précédemment dans une approche contextuelle adaptée aux vignobles des pays partenaires : Bulgarie, France, Italie, Royaume-Uni.

Le projet ECOWINERY [2] www.ecowinery.eu a pour objectif de fournir des outils méthodologiques pour l'écoconception de bâtiments vinicoles [3] aux viticulteurs, œnologues, responsables de domaines, techniciens conseil, architectes ou encore enseignants et étudiants en viticulture et œnologie (formation initiale et continue).

La construction d'un bâtiment vinicole et le choix des équipements associés à la conception de l'ouvrage supposent une réflexion approfondie concernant notamment les aspects fonctionnels. Au-delà, la prise en compte du développement durable impose une analyse relative à l'impact de la conception et du fonctionnement des caves sur l'environnement. Les caves anciennes, construites avant l'utilisation de la climatisation, intégraient des formes d'architecture traditionnelle qui permettaient naturellement de bénéficier de la fraîcheur en été et de la chaleur en hiver grâce notamment à l'utilisation de l'inertie thermique du sous-sol et de murs en pierre très épais qui atténuaient la variabilité thermique interne. La rénovation et la création de nouvelles caves, qui associent une bonne isolation éventuellement complétée de solutions originales (toits ou murs végétalisés), et des énergies alternatives (solaire, géothermie, biomasse), s'intègrent également dans cette dynamique de conception écologique.

En complément, une gestion rationnelle de cette eau doit être envisagée pour limiter les pertes inutiles et faciliter le traitement d'épuration, dont le dimensionnement et les coûts de fonctionnement sont étroitement liés au volume à traiter. Cet objectif justifie d'une part une optimisation de la conception des chais et d'autre part l'utilisation de méthodes de nettoyage et de désinfection appropriées.

Le traitement des effluents s'intègre également dans l'éco-conception d'une cave. L'impératif environnemental suppose de mettre en place un système de traitement individuel ou collectif performant. En complément, une vision durable intègre une réduction des coûts énergétiques liés à ce traitement ainsi qu'une diminution des déchets (boues) issus du processus d'épuration, avec si possible une bonne intégration paysagère.

Basée sur l'expérience des partenaires (Allemagne, Espagne, France Suisse), le projet ECOWINERY, l'outil E-Learning présente les différents aspects d'une écoconception d'une cave dans 4 modules :

- Module 1 : Enjeux environnementaux et contexte réglementaire (Fig. 2).

- Module 2 : Démarche architecturale et éco-construction : ressource énergétique associée au bâtiment (géothermie, solaire, pompe à chaleur, toit ou mur végétalisé) et éventuellement valorisation thermique.

\section{Water ressource distribution}

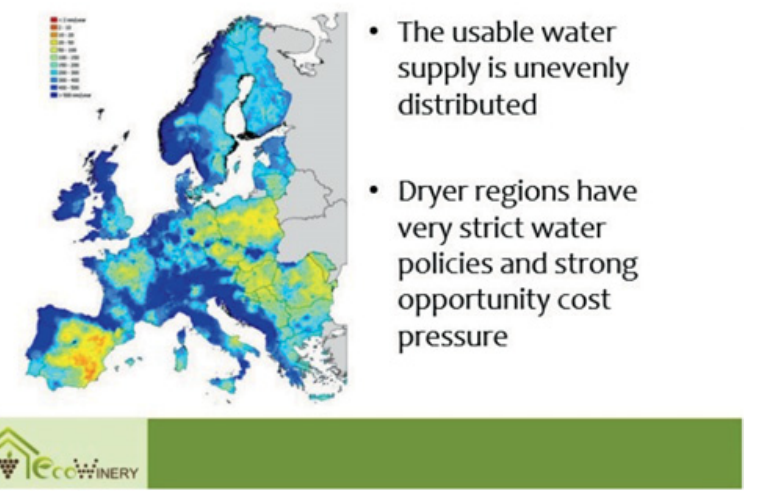

Figure 2. Exemple de présentation didactique du projet Ecowinery (carte de disponibilité en eau en Europe).

- Module 3 : Ressource énergétique associée au bâtiment (géothermie, solaire, pompe à chaleur, toit ou mur végétalisé) et la valorisation thermique.

- Module 4 : Optimisation de l'utilisation de l'eau dans une cave vinicole, économie et gestion écologique des effluents.

Un dernier module présente les aspects concrets de la réalisation d'une cave : Suivi et évaluation du projet d'écoconception d'un chai et de son impact environnemental. L'ensemble des modules est illustré de schémas, exemples, retour d'expériences de cas concrets caves « écologiques 》 dans le monde.

\section{Conclusion}

Cette méthode pédagogique permet de toucher un large public sans contraintes logistiques (faible empreinte carbone) et assure les différentes fonctions de l'enseignement dans un même outil : évaluation de l'apprenant, transfert de connaissances personnalisé (adaptation du niveau du contenu par interactivité). Ce processus assure une flexibilité de la gestion du temps de formation, notamment en situation de télétravail, avec la possibilité de moduler à son rythme, l'acquisition des connaissances. L'apprenant peut éventuellement acquérir des ressources et formation provenant d'experts par des documents, interviews difficiles à utiliser dans la formation classique. Par ailleurs la traduction en différentes langues des modules permet de toucher un public à l'échelle mondiale. En complément il est possible d'adapter l'ensemble de la formation ou certains modules à un contexte spécifique (local, technique etc.).

Ce type de formation présente néanmoins quelques contraintes liées notamment à la motivation et l'assiduité de l'apprenant qui peut justifier un contrôle de la progression distance par un tuteur. En effet, les apprenants doivent faire preuve de rigueur et de discipline, particulièrement s'ils sont isolés dans une formation à distance. Bien évidemment cette application nécessite la possibilité d'accéder à Internet avec une assez bonne maîtrise des outils informatiques. 


\section{Références}

[1] J. Rochard, I. Pierot, Congrès international de la vigne et du vin - Izmir, Turquie, Adaptation aux changements climatiques, projet européen Leonardo Da Vinci : E-VitiClimate (2013)

[2] J. Rochard, S. Penavayre et coll. Congrès Internationalde la Vigne et du Vin - Mendoza,
Argentine, Eco-conception des caves : du concept à la pratique, Projet européen Ecowinery (2014)

[3] J. Rochard. Revue des œnologues et des techniques vitivinicoles et œnologiques, Eco-construction et développement durable, ISSN 0760-9868, 36, Extra 133, pages. 11-12 (2009) 